\title{
SECUENCIAS DE FORRAJEO EN MONOS CAPUCHINOS EN CAUTIVERIO: APRENDIZAJE Y MEMORIA EN CONTEXTO DE GRUPO
}

\author{
FORAGING SEQUENCES IN CAPTIVE CAPUCHIN MONKEYS: \\ LEARNING AND MEMORY IN GROUP CONTEXT
}

MARÍA PAULA TUJAGUE 1234 Y HÉCTOR BLAS LAHITTE 25

${ }^{1}$ CONSEJO NACIONAL DE INVESTIGACIONES CIENTÍFICAS Y TÉCNICAS (CONICET, ARGENTINA)

\author{
${ }^{2}$ FACULTAD DE CIENCIAS NATURALES Y MUSEO, UNIVERSIDAD \\ NACIONAL DE LA PLATA (UNLP, ARGENTINA) \\ ${ }^{3}$ INSTITUTO DE BIOLOGÍA SUBTROPICAL, FACULTAD DE CIENCIAS \\ FORESTALES, UNIVERSIDAD NACIONAL DE MISIONES \\ ${ }^{4}$ ASOCIACIÓN CIVIL CENTRO DE INVESTIGACIONES DEL BOSQUE \\ ATLÁNTICO (CeIBA), PUERTO IGUAZÚ, MISIONES, ARGENTINA
}

${ }^{5}$ ACADEMIA NACIONAL DE CIENCIAS, ARGENTINA

\begin{abstract}
Resumen
Los primates diurnos son forrajeadores sociales que buscan el alimento en contexto de grupo. El objetivo del presente trabajo fue analizar las secuencias de desplazamiento durante la búsqueda de alimento de un grupo de monos capuchinos (Cebus libidinosus, Sapajus cay) en el Jardín Zoológico y Botánico de La Plata, utilizando el modelo esto-

Agradecemos al Jardín Zoológico y Botánico de La Plata por los permisos, a Verónica Trapani y Roberto Alonso por su asistencia y a María de los Ángeles Bacigalupe por sus comentarios sobre el manuscrito.

Esta investigación fue financiada por dos becas de UNLP y CONICET otorgadas a M. P. Tujague. El primer autor trabajó en la colecta de datos y ambos autores trabajaron en el diseño metodológico, el análisis y la preparación del manuscrito.

Correspondencia a María Paula Tujague, Departamento Científico de Etnografía, Museo de La Plata (Facultad de Ciencias Naturales y Museo, UNLP), Paseo del Bosque s/n, La Plata, Argentina. Correos electrónicos: mptujague@gmail.com,mptujague@conicet.gov.ar
\end{abstract}


cástico de aprendizaje como marco de referencia. Se instaló un juego de seis contenedores con comida accesible o inaccesible en el recinto de los individuos y se realizaron dos períodos experimentales, separados por un intervalo de tres meses. Se representaron las secuencias de visita a los contenedores y se construyó un código gráfico que permitiera la comparación entre secuencias. Se encontró una prevalencia de secuencias que involucraba la visita a un contenedor en ambos períodos y una disminución de las secuencias de tres contenedores durante el segundo periodo experimental, mientras que las secuencias de dos contenedores se mantuvieron constantes. Los resultados permiten inferir que los sujetos desarrollaron patrones de aprendizaje y memoria de lugar, optimizando las estrategias de forrajeo.

Palabras clave: monos capuchinos, secuencias de forrajeo, memoria, aprendizaje

\begin{abstract}
Diurnal primates are social foragers that search for resources as members of a social group. The aim of the present study was to analyze movement sequences in nine Capuchin monkeys (Cebus libidinosus, Sapajus cay) during foraging, using the stochastic learning model as a frame of reference. An array of six feeding sites was used, and each feeding site consisted of a container with either accessible or non accessible food. The movement sequences across the feeding sites were analyzed during two observation periods. Each period consisted of 15 sessions, and the two periods were separated by three months. A graphic code was designed to record the movement sequences. There was a prevalence of sequences involving visits to one feeder in both observation periods, and a decrease of sequences involving visits to three feeders in the second observation period, while sequences of visits to two feeders remained constant. We were able to determine changes in foraging sequence patterns which resulted in foraging efficiency.

Keywords: capuchin monkeys, foraging sequences, memory, learning.

Prácticamente todas las especies de primates diurnos son forrajeadores sociales que viajan y buscan los recursos alimenticios como miembros de un grupo (BiccaMarques \& Garber, 2005). Convivir con otros dentro de un grupo social puede ser de gran ventaja a la hora de aprender cuándo, cómo y qué comer (Visalberghi \& Addessi, 2001). El forrajeo (definido como la búsqueda, preparación y consumo del alimento) es uno de los ejemplos mejor conocidos sobre comportamientos modulados por la vida grupal (Boyer, Miramontes, Ramos-Fernández, Mateos, \& Cocho, 2003). Las actividades individuales de forrajeo pueden ser influenciadas por las actividades del resto de los miembros del grupo que se encuentren alimentándose en el mismo lugar (Bonnie \& de Waal, 2007), a la vez que los individuos pueden apoyarse en información social además de ecológica para localizar sitios de alimentación (Bicca-Marques \& Garber, 2005).
\end{abstract}


El concepto de búsqueda puede ser definido como el problema de qué hacer en situaciones que requieren explorar múltiples alternativas. La naturaleza serial de la búsqueda está implícita en su misma definición dado que implica la exploración de más de dos alternativas disponibles. Esta exploración serial requiere de la habilidad para registrar y recordar los movimientos realizados con el objeto de evitar la pérdida de tiempo y energía en reconsiderar alternativas ya exploradas por el mismo individuo o por otros miembros de su grupo (De Lillo, Visalberghi, \& Aversano, 1997). Puede sugerirse que, cuando los individuos están utilizando la memoria para evitar los sitios de alimento ya agotados o previamente visitados, los movimientos en el espacio serán irregulares. La memoria de sitios agotados y no agotados (Olton, 1982 en Cabrera, 2009) es la clásica alternativa de las tareas que pueden ser resueltas por medio del uso de estrategias que implican aprendizaje y memoria (Olton \& Samuelson,1976 en Yoerg \& Kamil, 1982).

Según la teoría del aprendizaje social, la información que un sujeto puede obtener por medio de la observación no proviene solo de su propio comportamiento sino también de las consecuencias de los comportamientos de un modelador o compañero de grupo (Bandura, 1984). Podemos distinguir dos tipos de aprendizaje social (Heyes, 1993): el aprendizaje social imitativo (imitación) y el aprendizaje social no imitativo. El aprendizaje social imitativo es definido como el aprendizaje a través de la observación o interacción directa con un conespecífico o compañero de grupo, o con los productos del comportamiento de éste (señales olfativas, ítems de alimento descubiertos, madrigueras, etc.). El aprendizaje social no imitativo, en cambio, comprende el aprendizaje sobre el ambiente (estímulos, objetos y eventos) a través de la observación ya sea para diferenciar a estos estímulos de otros tipos posibles o para relacionarlos con un valor positivo o negativo en virtud de su relación con objetos y eventos. El presente estudio evalúa ambos tipos de aprendizaje: el aprendizaje social no imitativo de objetos (un dispositivo experimental) y eventos (la presencia de comida accesible e inaccesible) en contexto de grupo, permitiendo a los individuos observar las actividades del resto de los individuos (aprendizaje social imitativo), utilizando el modelo de aprendizaje estocástico como marco teórico.

Los modelos estocásticos fueron desarrollados en sus inicios por Bush \& Mosteller (1951, 1955 en Santisteban Requena, 2000) y consistían de modelos matemáticos basados en la fuerza de la respuesta durante el aprendizaje (Santisteban Requena, 2000). Las elecciones sucesivas durante el proceso de aprendizaje se correlacionan unas con otras, y cada refuerzo positivo incrementa la probabilidad de respuesta en una cantidad que es una fracción constante de la diferencia entre la probabilidad actual y la probabilidad máxima de ser recompensado, mientras que la ausencia de refuerzo disminuye la probabilidad de respuesta en una cantidad que es una fracción constante de la diferencia entre la probabilidad actual y la probabilidad mínima de ser recompensado (Staddon \& Horner, 1989). 
El modelo de aprendizaje estocástico (Lahitte, 1992) explica los resultados de un proceso de aprendizaje por medio de la utilización de un modelo teórico construido desde el análisis de una situación experimental, explicando la dependencia de secuencias entre situación estímulo - respuesta del sujeto - refuerzo. El modelo incluye: 1) el análisis de la situación (descripción), 2) la codificación de la situación experimental para analizar el comportamiento y 3) una descripción del marco teórico. El objetivo de estos modelos es explicar una experiencia de observación mediante la extracción de rasgos distintivos y la construcción de un código, ambos relacionados a hipótesis previamente establecidas. Los modelos estocásticos centran su atención en la dependencia secuencial de los fenómenos y siempre incluyen los siguientes elementos:

1 - La situación experimental. El sujeto es incluido en una serie de experiencias y se pueden discriminar tres eventos de observación en cada una de esas experiencias:

$\mathrm{E}=$ conjunto de posibles situaciones/estímulo que son presentados por el investigador.

Rs $=$ conjunto de posibles respuestas ejecutadas por el sujeto.

$\mathrm{Re}=$ conjunto de posibles refuerzos presentados por el investigador.

2 - Las características del sujeto incluido en la situación experimental.

3 - Las experiencias previas del sujeto incluido en la situación experimental.

4 - Las reglas experimentales decididas por el experimentador. Se entienden como reglas experimentales a la manera en que la situación experimental es construida junto con las reglas de finalización (la decisión sobre el momento en que el experimento terminal.

El conjunto de posibles respuestas son analizadas sobre la base de la previa codificación del comportamiento. Podemos definir los cambios entre los sujetos, y entre ellos y el contexto experimental, a través de la codificación de secuencias de acciones y su repetición y redundancia. Por lo tanto, la repetición y redundancia de las scuecias de forrajeo es la mejor manera de detectar cambios comportamentales.

Los estudios en cautiverio realizados hasta el momento para evaluar la memoria espacial de los capuchinos incluyeron sólo investigaciones que prueban a los individuos aislados de su grupo, una situación totalmente opuesta a lo que ocurre en vida silvestre donde este comportamiento ha evolucionado (Fragaszy, Johnson Pynn, Hirsh, \& Brakke, 2003; D Amato \& Buckiewicz, 1980 en Fragaszy, Visalberghi, \& Fedigan, 2004; Potì, 2000; Potì, Bartolommei, \& Saparoti, 2005; Tavares \& Tomaz, 2000; Spinozzi, Lubrano, \& Truppa, 2002; Rosengart \& Fragaszy, 2003).

El objetivo del presente trabajo fue determinar los patrones de aprendizaje serial y memoria en los monos caí o capuchinos (Cebus libidinosus = Sapajus cay) por medio de la observación, el registro y la descripción de secuencias de movimiento durante el forrajeo. Se enfocó el estudio en la descripción de los patrones de búsqueda de los sujetos experimentales para evaluar la hipótesis acerca de que los monos realizan las visitas a sitios con comida en patrones de grupo de manera progresiva y que esos patrones influyen sobre el desarrollo de la memoria para maximizar su eficiencia de forrajeo. 
Se trabajó con un diseño cuasi-experimental para evaluar la memoria espacial en un ambiente fijo y en su contexto grupal normal. El problema al cual fueron expuestos los individuos fue dónde la comida era accesible y dónde no. La organización espontánea del comportamiento en la ausencia de un entrenamiento explícito fue evaluada por medio de la disminución en el número de movimientos durante la búsqueda.

\section{Método}

\section{Sujetos}

Para el presente estudio se evaluó un grupo de monos capuchinos $(\mathrm{N}=9)$ alojados en un recinto-jaula de materiales convencionales en el Jardín Zoológico y Botánico de La Plata, Buenos Aires, Argentina. Todos los miembros del grupo fueron identificados por el tamaño corporal, patrón de coloración y marcas faciales distintivas. La composición del grupo fue la siguiente: Tres adultos de seis años de edad o mayores (dos machos y una hembra) identificados con los nombres de GOR, $\mathrm{CH}$ y CE respectivamente, y seis monos juveniles de uno a cinco años de edad (cuatro machos y dos hembras) identificados con los nombres de MN, NI, TO, PU, DIE y RO respectivamente. Los sujetos sólo habían participado en programas de enriquecimiento ambiental a cargo de los cuidadores de la institución.

El grupo fue alimentado una vez al día con una dieta compleja que consistía en vegetales, frutas, huevos, cereales y trozos de pollo cocido. El alimento proporcionado durante el experimento fue integrado a la dieta normal de los individuos para evitar que ganaran peso en exceso.

\section{Materiales y diseño experimental}

El experimento se llevó a cabo en la misma jaula donde los capuchinos se alojaban $(14,80 \mathrm{~m} \times 4 \mathrm{~m})$. La jaula estaba dividida en dos recintos interiores A y B (cada uno de ellos con un tamaño de $6 \mathrm{~m} \times 4 \mathrm{~m}$ ) separados por una puerta guillotina. A cada lado de los recintos A y B se ubicaba un recinto para dormir de 2,80 m x $4 \mathrm{~m}$. El experimento se desarrolló siempre en el recinto $A$ donde los monos recibían la comida de manera habitual.

El diseño experimental utilizado se basó en una versión modificada del esquema utilizado por Tarou y Maple (2000) y por Ludvig, Tang, Eichenbaum y Gohil (2003). El objetivo del mismo fue estimular a los monos a interactuar libremente con localizaciones de alimento en un espacio tridimensional, considerando su comportamiento natural de forrajeo (Sánchez- Vazquez, Lahitte, \& Tujague, 2011).

Los monos se movían recolectando comida de un conjunto de tres contenedores con comida accesible (CA) intercalados entre tres contenedores con comida 
inaccesible (CI). Los contenedores estaban construidos de PVC (polyvinyl chloride) y colgados de dos mallas de alambre ubicadas de manera opuesta y en una posición equidistante unos de otros dentro de la jaula, atados con una soga e imposibles de mover de su sitio por acción de los individuos. Si bien existían numerosas señales externas disponibles para los individuos, los contenedores eran idénticos en forma (cilíndricos), tamaño (14 cm de altura y $11 \mathrm{~cm}$ de diámetro) y color (blanco opaco), y todos ellos llevaban comida en su interior (copos de maíz sin azúcar) imposible de visualizar externamente, para evitar que existieran diferencias olfativas entre ellos (ver Figura 1).

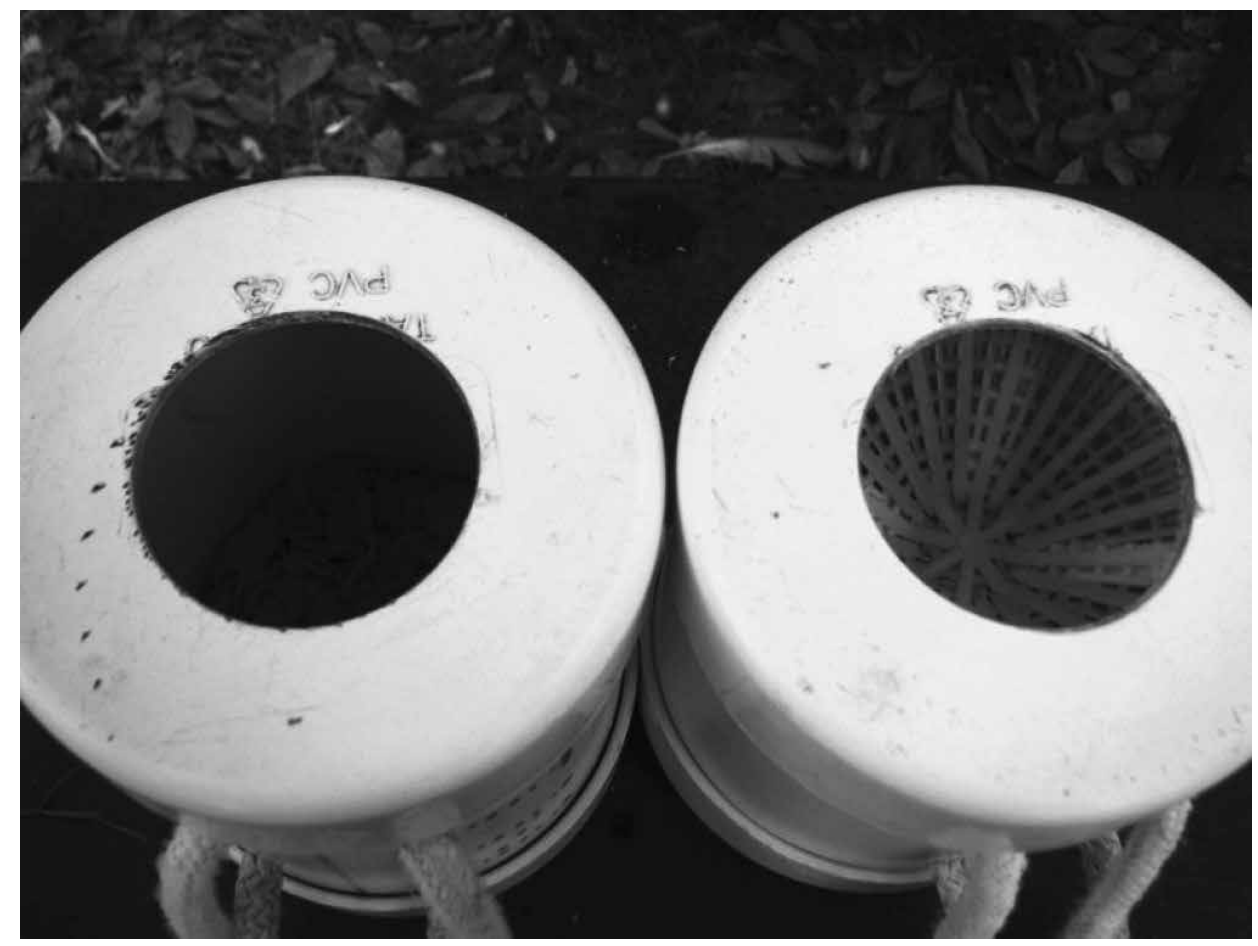

Figura 1. Contenedores accesibles e inaccesibles.

Los capuchinos podían insertar sus manos en un agujero en la parte superior de los contenedores accesibles para extraer los ítems de comida. Los contenedores inaccesibles llevaban por dentro una malla plástica de forma cónica que hacía posible insertar la mano pero imposible acceder a la comida. La única manera en que los individuos podían revisar si un contenedor tenía comida accesible era insertando su mano en el agujero o mirando a través del mismo. Para simplificar la recolección de datos los contendores fueron numerados del 1 a 6 , siendo 1,3 y 5 los contenedores accesibles, y 2, 4 y 6 los inaccesibles. 
Los monos se encontraban por lo menos con 20 horas de ayuno previo a cada sesión experimental pero tenían acceso libre al agua para beber. Dos asistentes ayudaron en la instalación de los contenedores experimentales en la jaula para cada sesión de trabajo y los retiraron al final las mismas.

\section{Procedimiento}

Utilizando como marco de referencia al modelo de aprendizaje estocástico se codificó la situación experimental de la siguiente manera:

$\mathrm{E}=(\mathrm{e} 1, \mathrm{e} 2, \mathrm{e} 3, \mathrm{e} 4, \mathrm{e} 5, \mathrm{e})$ siendo e1 a e6 la presencia de los seis contenedores de comida.

$\mathrm{Rs}=(\mathrm{rs} 1, \mathrm{rs} 2)$ siendo rs1 la visita a un contenedor accesible y rs2 la visita a un contenedor no accesible.

$\mathrm{Re}=(\mathrm{re} 1, \mathrm{re} 2)$ siendo re1 la presencia de comida accesible en un contenedor accesible y $\mathrm{r} 2$ la ausencia de comida accesible en un contenedor inaccesible.

Los contenedores fueron Ilenados e instalados antes del inicio de cada sesión en ausencia de los sujetos experimentales. Durante este procedimiento los individuos fueron aislados en el recinto $B$, y solo se les permitió el ingreso al recinto $A$ después de que los contendores ya estaban instalados en sus posiciones asignadas. Las sesiones se llevaron a cabo por la mañana antes del horario de alimentación fijado por el zoológico. Se consideró sesión experimental a cada oportunidad en la que los monos fueron expuestos al diseño experimental (Sánchez Vazquez et al., 2011).

Se registraron los patrones de visita a los contenedores y la identidad de cada individuo involucrado en la visita registrada. Se definió como evento de visita a cada vez que los monos se acercaban a un contenedor (con comida accesible o inaccesible) para mirar en su interior, realizar comportamientos exploratorios o retirar alimento (ver Figura 2). En el experimento se consideró que había comportamiento exploratorio en cada oportunidad en la que los monos pusieron sus manos y/o pies sobre un contenedor, empujándolo hacia arriba o abajo, golpeándolo o intentando abrirlo con la boca (Sánchez Vazquez et al., 2011).

Se evaluó el comportamiento de los monos de la siguiente manera:

1 - Período de habituación: Se instalaron dos contenedores con comida accesible, dejando que los individuos interactuaran con ellos. El criterio de finalización del periodo de habituación fue cuando cada individuo había retirado comida del interior de un contenedor al menos una vez. Esto ocurrió a los cinco días después de la primera exposición a los contenedores. Los individuos empezaron a buscar en los contendores apenas se los dejó acceder a los mismos dentro del recinto A.

2 - Primer período experimental de aprendizaje y memoria (PE1): después del periodo de habituación se evaluó el comportamiento de los monos en el primer período experimental que consistió de 15 sesiones de exposición al diseño de seis 


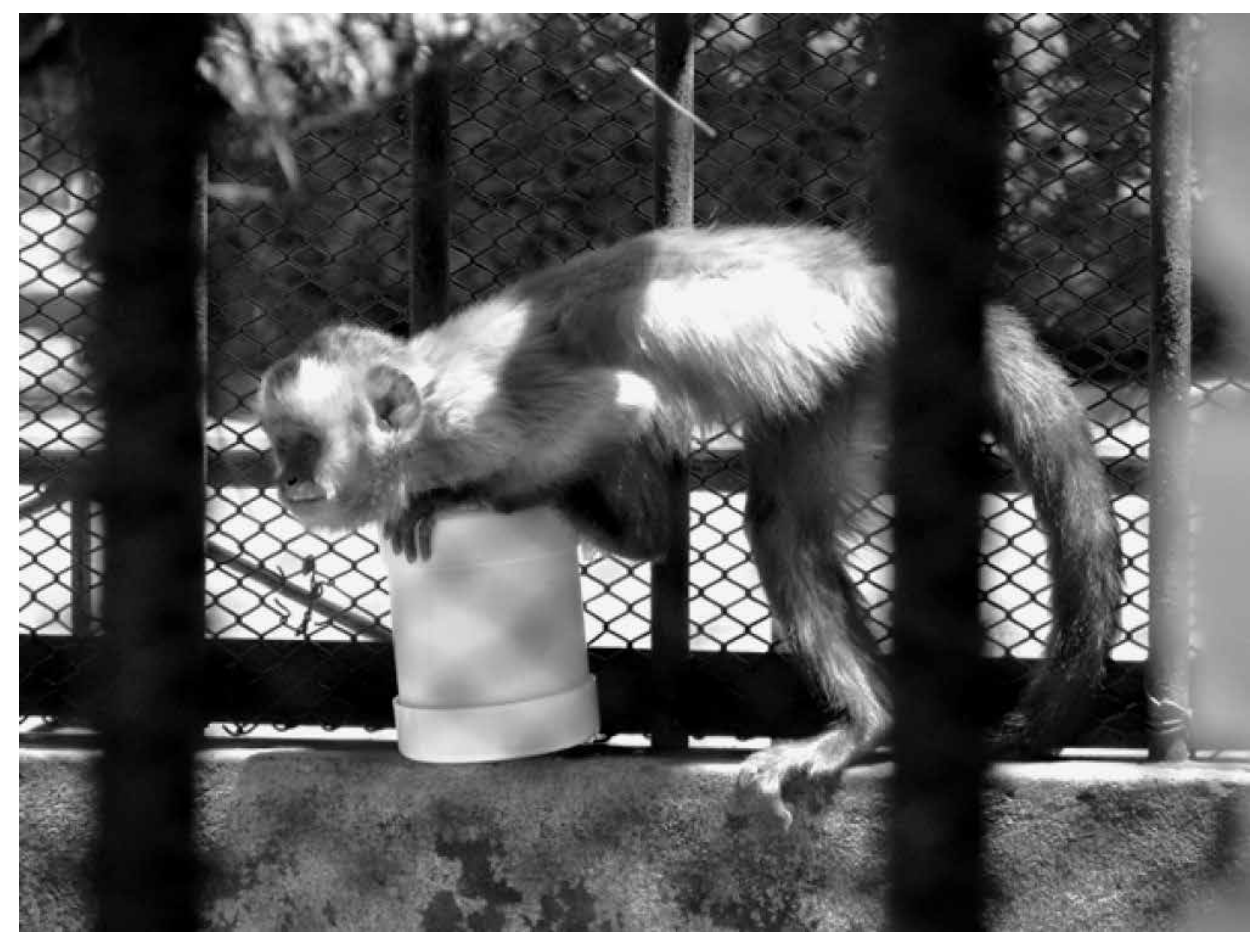

Figura 2. Juvenil visitando un contenedor accesible.

contenedores descrito con anterioridad. Cada sesión tuvo lugar transcurridas las 48 horas de la sesión anterior.

3 - Segundo período experimental de aprendizaje y memoria (PE2): después de transcurrido un periodo de tres meses sin exponer a los individuos al diseño experimental, se repitió el mismo esquema que en PE1.

A lo largo de las sesiones de PE1 y PE2 se evaluó la habilidad de los monos para aprender y recordar la posición espacial de los contenedores y optimizar su eficiencia de forrajeo. Las visitas a contenedores accesibles (CA) e inaccesibles (Cl) se registraron por medio de la técnica de muestreo de Animal focal de 10 minutos de duración en PE1 y muestreo de Grupo focal en PE2 (Altman, 1974). La razón de esta diferencia en los métodos de muestreo para cada período experimental dependió de la mayor habilidad para reconocer la identidad de los sujetos en el segundo período experimental, lo que permitió al observador registrar los comportamientos de todo el grupo al mismo tiempo. Ambos métodos son clásicos en el estudio del comportamiento animal, y permiten a los investigadores recolectar datos sin interferir con la situación ambiental de los sujetos. Se consideró como una visita correcta (VC) cuando los monos retiraban comida de un contenedor con comida accesible, e incorrecta 


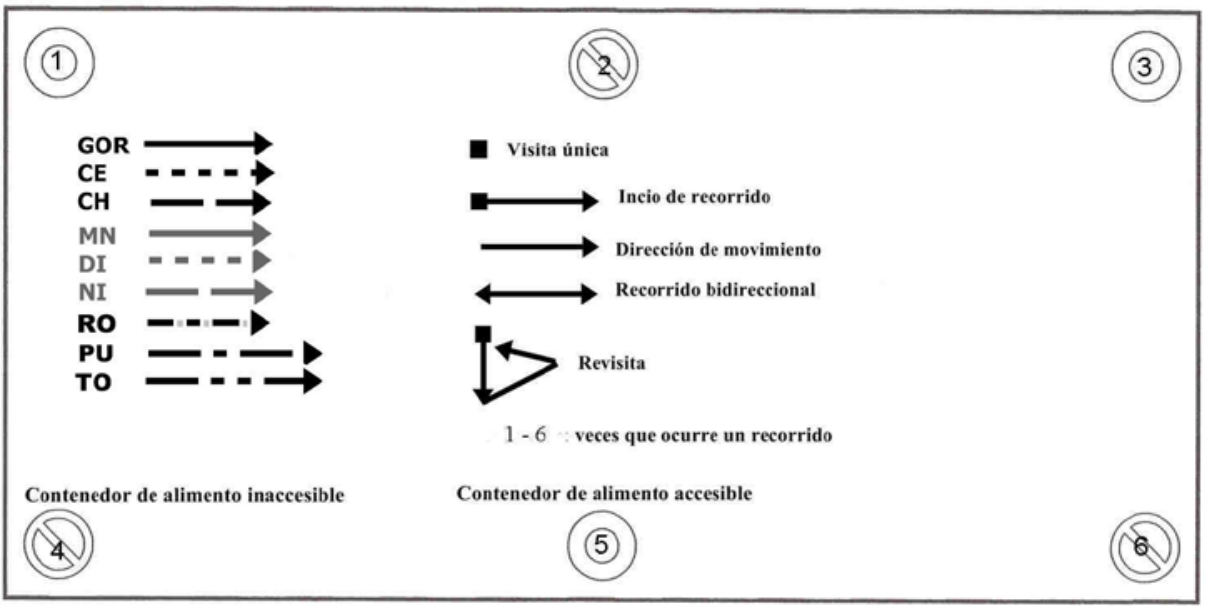

Figura 3. Código gráfico para representar las secuencias de movimiento de los individuos.

(VI) si los monos trata-ban de retirar comida o se acercaban a un contenedor con comida inaccesible.

Cada sesión experimental inició con los monos entrando al recinto A y finalizó cuando al menos uno de los contendores CA estuviera vacío. Las sesiones duraron en promedio 62 minutos para PE1 $(D E=15.19)$ y 43,6 minutos para PE2 $(D E=10.7)$.

Las variables cuantitativas analizadas fueron el número de VC y el número de VI, mientras que las variables cualitativas analizadas fueron los patrones de forrajeo (secuencias repetitivas de visitas que los sujetos realizaron a lo largo del experimento) y estrategias de comportamiento (comportamientos desarrollados a partir de la interacción con el diseño experimental).

Con el objetivo de representar a los individuos y sus visitas a los contenedores se creó un código gráfico (Figura 3). Utilizando este código y usando el programa Photo Scape 3.5 (software libre) se graficaron las secuencias de movimiento para obtener así un mapa de secuencias para cada sesión.

La codificación de secuencias fue construida a partir del análisis gráfico de los movimientos de los monos entre los contendores 1 a 6 . Una vez que se concluyó la codificación se procedió a detectar de manera visual la repetición y redundancia de secuencias, e identificar cambios entre sesiones a nivel grupal.

Nota ética: todas las investigaciones realizadas en este manuscrito adhirieron a los principios éticos para el tratamiento de primates no humanos de la American Society of Primatologists y a los protocolos aprobados por el Animal Care and Use Committee. También adhirió a los requerimientos legales de Argentina y fue aprobado por el Jardín Zoológico y Botánico de La Plata. 


\section{Resultados}

Se obtuvieron 30 mapas correspondientes a las 30 sesiones experimentales totales (PE1 y PE2). Las sesiones de habituación fueron excluidas del análisis.

Como resultado de la evaluación gráfica se identificaron secuencias de visita a los contenedores que fueron codificadas de la siguiente manera: seis visitas a un solo contenedor (contenedor único) accesible o inaccesible, 10 secuencias de visita a dos contenedores (Tabla 1) y 25 secuencias de visita a tres contenedores (Tabla 2).

Como puede observarse en la Tabla 1, no hubieron registros de secuencias de dos contenedores que incluyeran contenedores inaccesibles. A su vez, las secuencias de tres contenedores codificadas como $3 \mathrm{~S} \mathrm{CA} \mathrm{o} \mathrm{Cl}$ solo incluyeron contenedores accesibles.

Se muestra a modo de ejemplo un mapa de secuencias del período PE2 (Figura 4). Las secuencias de contenedor único no fueron representadas en el mapa.

Tabla 1

Descripción de secuencias de dos contenedores.

\begin{tabular}{cc}
\hline \multicolumn{2}{c}{ Secuencia de contenedores } \\
\hline Tipo de contenedor & Contenedor involucrado \\
\hline 2 CA & $3-1$ \\
& $5-5$ \\
& $1-5$ \\
& $3-1$ ó $1-3$ \\
& $3-5$ ó $5-3$ \\
& $1-4$ \\
$2 \mathrm{CA}+\mathrm{Cl}$ & $3-2$ \\
& $4-5$ ó $5-4$ \\
& $5-6$ ó 6-5 \\
\hline
\end{tabular}

Nota. 2 AC: secuencia de 2 contenedores accesibles; $2 \mathrm{CA}+\mathrm{Cl}$ : secuencias que incluyen 1 contenedor accesible más 1 contenedor inaccesible. 
Tabla 2

Descripción de secuencias de tres contenedores

\begin{tabular}{|c|c|}
\hline \multicolumn{2}{|c|}{ Secuencia de contenedores } \\
\hline Tipo de contenedor & Contenedor involucrado \\
\hline Simple CA o Cl & 1-3-1 ó 3-1-3 \\
\hline \multirow[t]{2}{*}{$(3 \mathrm{~S} C A \circ \mathrm{Cl})$} & 1-5-1 ó 5-1-5 \\
\hline & 3-5-3 ó 5-3-5 \\
\hline Simple $\mathrm{CA}+\mathrm{Cl}$ & $3-2-3$ \\
\hline \multirow[t]{5}{*}{$(3 \mathrm{~S} C A+C I)$} & $4-1-4$ \\
\hline & 1-6-1 ó 6-1-6 \\
\hline & 3-6-3 ó 6-3-6 \\
\hline & 4-5-4 ó 5-4-5 \\
\hline & 5-6-5 ó 6-5-6 \\
\hline Compleja CA o Cl & $4-2-6$ \\
\hline$(3 \mathrm{C} \mathrm{CA} \mathrm{o} \mathrm{Cl})$ & 1-5-3 ó 5-1-3 ó 1-3-5 ó 3-1-5 ó 5-3-1 \\
\hline Compleja $\mathrm{CA}+\mathrm{Cl}$ & $3-2-4$ \\
\hline \multirow[t]{7}{*}{$(3 \mathrm{C} \mathrm{CA}+\mathrm{Cl})$} & $1-4-6$ o $6-1-4$ \\
\hline & $2-1-5$ o $5-1-2$ \\
\hline & $3-1-4$ o $1-3-4$ o $4-1-3$ \\
\hline & $5-4-3$ o $4-5-3$ o $3-4-5$ \\
\hline & $6-2-1$ o $2-1-6$ o $6-1-2$ \\
\hline & $6-2-3$ o $2-6-3$ o $3-2-6$ \\
\hline & $1-6-5$ o $1-5-6$ o $6-1-5$ o 5-6-1 \\
\hline
\end{tabular}


Secuencia de contenedores

\begin{tabular}{|c|c|}
\hline \multirow[t]{7}{*}{ Tipo de contenedor } & Contenedor involucrado \\
\hline & $3-1-6$ o $1-6-3$ o $6-3-1$ o $3-6-1$ \\
\hline & $3-2-1$ o $1-3-2$ o $2-3-1$ o $1-2-3$ \\
\hline & $5-4-1$ o $5-1-4$ o $1-4-5$ o $4-5-1$ \\
\hline & $6-5-4$ o 5-6-4 о 4-6-5 о 4-5-6 \\
\hline & $3-6-5$ o $5-3-6$ o $3-5-6$ o 5-6-3 о 6-5-3 \\
\hline & $3-2-5$ o $5-3-2$ o $2-3-5$ o $2-5-3$ о $5-2-3$ \\
\hline
\end{tabular}

Nota. Secuencia simple: 2 de los 3 contenedores son el mismo que es visitado 2 veces. Secuencia compleja: los 3 contenedores son diferentes. Simple CA o Cl (3S CA o Cl): secuencias simples de 3 contenedores que incluyen contenedores accesibles o inaccesibles solamente y 2 de esos 3 contenedores son el mismo que es visitado 2 veces; Simple $\mathrm{CA}+\mathrm{Cl}(3 \mathrm{~S} \mathrm{CA}+\mathrm{Cl})$ : secuencias simples de 3 contenedores que incluyen contenedores accesibles más inaccesibles y 2 de esos 3 contenedores son el mismo que es visitado 2 veces; Compleja $\mathrm{CA}$ o $\mathrm{Cl}$ (3C CA o Cl): secuencias complejas de 3 contenedores que incluyen 3 contenedores accesibles o inaccesibles diferentes; Compleja $\mathrm{CA}+\mathrm{Cl}(3 \mathrm{C} C \mathrm{C}+\mathrm{Cl})$ : secuencias complejas de 3 contenedores que incluyen contenedores accesibles más inaccesibles en la misma secuencia.

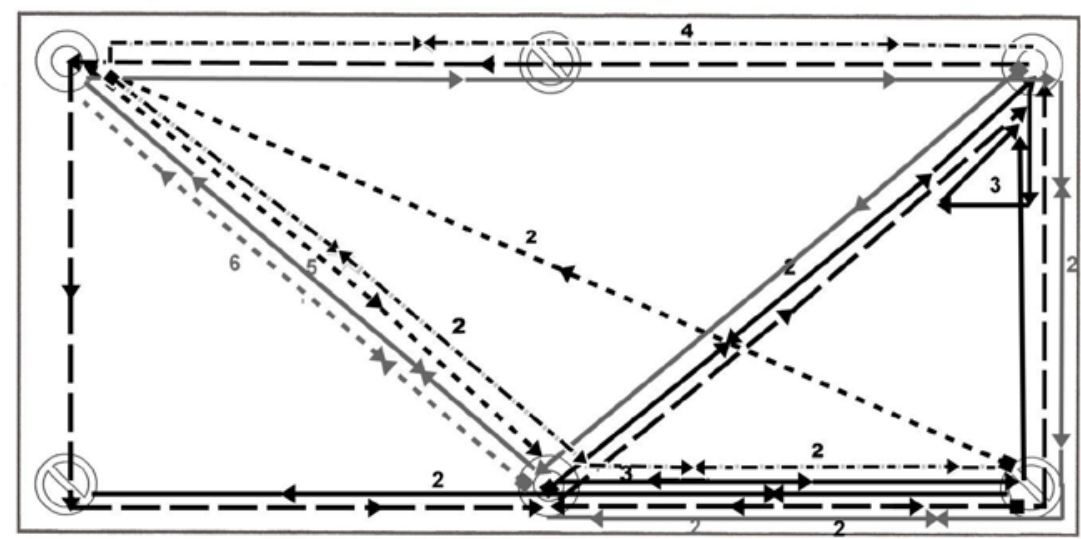

Figura 4. Ejemplo de un mapa de secuencias (sesión 5, período PE2). Los números indican cuántas veces fue ejecutada la secuencia. Cada secuencia representa un individuo diferente.

Posterior a la identificación y codificación de secuencias se analizaron las frecuencias de realización de las mismas en cada período experimental. Las secuencias de contenedor único accesible (CA) se observaron con mayor frecuencia en ambos 
períodos (38.9 \% para PE1 y 59.2 \% para PE2), mientras que la realización de secuencias de contenedor único inaccesible $(\mathrm{Cl})$ fue menor y disminuyó de $9.2 \%$ en PE1 a $1.2 \%$ en PE2.

La realización de secuencias de dos contenedores fue mayor en PE1 que en PE2. Las secuencias 2 CA disminuyeron de $4.6 \%$ a $2.4 \%$ mientras que las secuencias $2 \mathrm{CA}+\mathrm{Cl}$ de $4.6 \%$ a $1.0 \%$.

Se registró una disminución en la realización de secuencias simples y complejas de tres contenedores accesibles más inaccesibles $(3 \mathrm{~S} \mathrm{CA}+\mathrm{Cl}$ y $3 \mathrm{C} \mathrm{CA}+\mathrm{Cl})$. Su ocurrencia fue de 13.7 \% y 26 \% en PE1 y disminuyó a $3.7 \%$ y $15.4 \%$ respectivamente en PE2. Por otra parte se obtuvo lo opuesto para las secuencias simples y complejas de tres contenedores accesibles o inaccesibles ( $3 \mathrm{~S} \mathrm{CA} \mathrm{o} \mathrm{Cl}$ y $3 \mathrm{C} \mathrm{CA} \mathrm{o} \mathrm{Cl}$ ), que mostraron un incremento en su ocurrencia de $0.7 \%$ a $8.8 \%$ para $3 \mathrm{~S} \mathrm{CA} \mathrm{o} \mathrm{Cl}$ y $0.8 \%$ a $8.3 \%$ para $3 \mathrm{C} \mathrm{CA} \mathrm{o} \mathrm{Cl}$.

El registro de secuencias de contenedor único y de dos contenedores mostró los siguientes patrones: durante PE1 las secuencias de contenedor único CA mostraron valores promedios similares para adultos $(M=6, D E=2)$ y juveniles $(M=5, D E=1.5)$, mientras que en PE2 los juveniles mostraron valores elevados $(M=58.2, D E=6.4)$ excluyendo al individuo TO quien realizó el doble de secuencias que el resto del grupo)en comparación con los adultos $(M=37.7, D E=6.8)$. La realización de secuencias de contenedor único $\mathrm{Cl}$ tuvo valores bajos tanto para adultos como juveniles en ambos períodos experimentales (cero a tres ejecuciones por individuo exceptuando al individuo TO quien ejecutó cinco secuencias en PE2).

El registro de secuencias de dos contenedores accesibles durante PE1 y PE2 tuvo valores bajos para adultos y juveniles registrándose de 0 a 3 secuencias realizadas por individuo, exceptuando al individuo TO con 10 ejecuciones en PE2. El registro de secuencias $2 \mathrm{CA}+\mathrm{Cl}$ no mostró diferencias entre períodos siendo en ambos casos bajo (cero a dos ejecuciones por individuo exceptuando al individuo PU quien realizó cinco secuencias en PE2).

Para el caso de las secuencias de tres contenedores, simples o complejas $\mathrm{CA}$ o $\mathrm{Cl}$, no se registraron secuencias de este tipo en PE1 (con excepción del individuo RO con una sola realización de una secuencia $3 \mathrm{C} \mathrm{CA} \mathrm{o} \mathrm{Cl).} \mathrm{Sin} \mathrm{embargo} \mathrm{la} \mathrm{cantidad} \mathrm{de} \mathrm{registros} \mathrm{au-}$ mentó en PE2 mostrando un valor promedio de $9.3(D E=3.7)$ en juveniles y de $6(D E=$ 3.6) en adultos para las secuencias $3 \mathrm{~S} \mathrm{CA} \mathrm{o} \mathrm{Cl}$, y un valor promedio de $9.3(D E=2.7)$ en juveniles y $6.5(D E=2.1)$ en machos adultos para las secuencias $3 \mathrm{C} \mathrm{CA} \mathrm{o} \mathrm{Cl}$. La única hembra adulta, $C E$, no realizó secuencias de tres contenedores accesibles o inaccesibles.

Las secuencias de tres contenedores simples y complejas $\mathrm{CA}+\mathrm{Cl}$ no fueron realizadas por los adultos en PE1 mientras que los juveniles realizaron 17 secuencias simples y 32 complejas. Durante PE2 se registró un total de 31 y 129 secuencias simples y complejas respectivamente, con las siguientes diferencias en los valores promedio entre adultos y juveniles: En el caso de secuencias $3 \mathrm{~S} \mathrm{CA}+\mathrm{Cl}$, la media $(M)$ fue de $2.7(D E=1.5)$ para adultos y de $3.8(D E=2.4)$ para juveniles. En el caso 


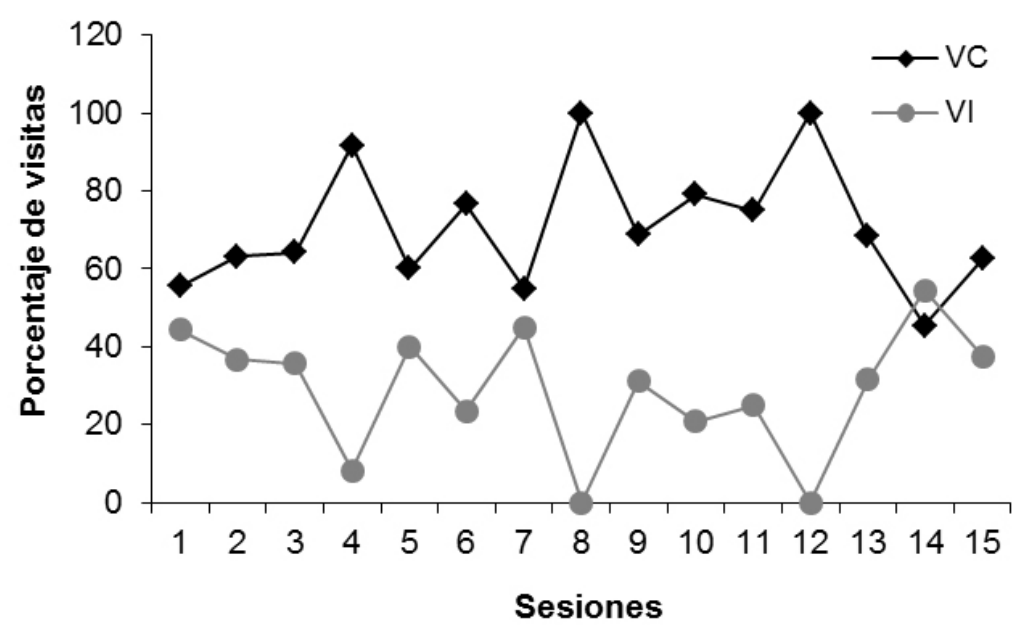

Figura 5. Porcentaje de Visitas Correctas (VC) y Visitas Incorrectas (VI) durante las sesiones experimentales del primer periodo de aprendizaje y memoria (PE1).

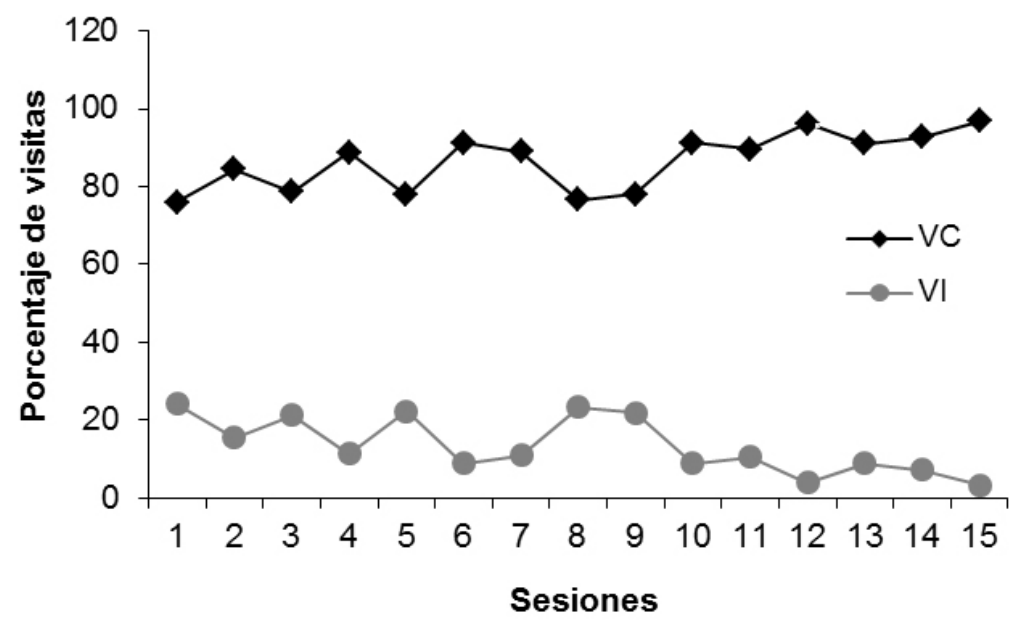

Figura 6. Porcentaje de Visitas Correctas (VC) y Visitas Incorrectas (VI) durante las sesiones experimentales del segundo período de aprendizaje y memoria (PE2). 
de secuencias $3 C \mathrm{CA}+\mathrm{Cl}$ la media fue de 13.3 para adultos $(D E=3.05)$ y de 14.8 $(\mathrm{DE}=8.7)$ para juveniles.

Los valores de visitas correctas e incorrectas durante las sesiones de PE1 (Figura 5) y PE2 (Figura 6) mostraron un patrón inestable durante PE1, con fluctuaciones de los valores a lo largo de las sesiones, mientras que durante PE2 se observó un patrón estable con un porcentaje alto de visitas correctas y bajo de incorrectas.

\section{Discusión}

Se encontró una prevalencia de secuencias de contenedor único durante ambos períodos de aprendizaje y memoria, con un incremento en el registro de secuencias que incluyeran contenedores accesibles (CA) y una disminución de secuencias que incluyeran contenedores inaccesibles (Cl) durante PE2.

El patrón observado para las secuencias de dos contenedores demostró la disminución en el número de visitas a contenedores inaccesibles. Por otra parte, las secuencias de contenedor único accesible presentaron valores elevados mientras que las secuencias de dos contenedores fueron bajas en ambos períodos. Finalmente la disminución de secuencias simples y complejas de tres contenedores $\mathrm{CA}+\mathrm{Cl}$ y la situación opuesta para las secuencias $\mathrm{CA}$ o $\mathrm{Cl}$ indican que los sujetos aprendieron a evitar los contenedores inaccesibles.

Sumado a lo descrito con anterioridad, la estabilización de los valores de visitas correctas e incorrectas durante PE2 en comparación con PE1, y la presencia de valores elevados de visitas correctas durante PE2 sugieren que los sujetos aprendieron las posiciones espaciales y los patrones de forrajeo que los llevaron a desarrollar memoria espacial para CA y Cl, ayudándolos a optimizar su eficiencia de forrajeo. El análisis de los patrones de comportamiento de los individuos reveló que los juveniles realizaron un número elevado de secuencias en comparación con los adultos, pero ambos (juveniles y adultos) demostraron secuencias con mayor cantidad de contenedores accesibles que inaccesibles.

Un resultado relevante encontrado en este trabajo es el incremento de las secuencias de tres contenedores en PE2, con valores similares para juveniles y adultos. Como Aparicio y Huidobro (1990) afirmaron, muchos mamíferos alternan entre localizaciones de alimento una vez que han aprendido las posiciones de la comida accesible. Puede inferirse que en la situación experimental planteada durante el presente experimento la actividad de forrajeo per se necesita incluir sitios inaccesibles para preservar la alternancia ambiental que ocurre en condiciones silvestres.

Se ha observado que la proximidad espacial entre individuos revela fuertes vínculos sociales entre ellos (Janson, 1990). Las fuertes preferencias espaciales han sido documentadas en casi todas las especies de primates estudiados tanto en cautiverio como en vida silvestre (Wilson, 1972 en Janson, 1990). Bonnie y de Waal (2007) hallaron que los capuchinos no necesitaban ser recompensados -e incluso, no necesitaban siquiera visualizar una recompensa - para ser influenciados por el comportamiento de forrajeo de otros individuos. Los compañeros sociales influenciaban las 
decisiones de forrajeo de otros miembros del grupo por medio de reforzar la relevancia de objetos (refuerzo de estímulos) o localizaciones (refuerzo de localizaciones) en el ambiente, solo por la recompensa intrínseca de actuar como los demás miembros del grupo (Modelo del aprendizaje observacional basado en la identificación y el vínculo (Bonnie \& de Waal, 2007).

El análisis de estrategias individuales permite detectar patrones generales de forrajeo (Alfaro, García-Leal, \& Cabrera, 2010). Utilizando la repetición y redundancia de secuencias codificadas por el observador se obtuvieron patrones de movimiento compartidos que los sujetos aprendieron en contexto grupal, reforzando las ideas explicadas con anterioridad. Es importante enfatizar la importancia de la habilidad del investigador para crear códigos que representen el comportamiento observado.

Como han postulado Visalberghi y Tomasello (1998) en Bicca-Marques y Garber (2005, p. 1341): "Much of the adaptive behaviour of primates depends on individual learning about recurrent sequences of events in their physical and social environments, and then exploiting the regularities in formulating their own behavioural strategies."

A lo largo del presente estudio se ha demostrado que el uso del modelo estocástico de aprendizaje permite al observador analizar una situación experimental obteniendo como resultado un código que ofrece a otros investigadores la posibilidad de utilizarlo para evaluar otros grupos de individuos en la misma situación experimental.

\section{Referencias}

Alfaro, L., García-Leal, O., \& Cabrera, R. (2010). Estrategias de búsqueda y consumo de alimento en grupos de ratas expuestos a diferentes distribuciones de alimento. Revista Mexicana de Anбlisis de la Conducta, 36, 93-109.

Altmann, J. (1974). Observational study of behaviour: sampling methods. Behaviour, 49, 227-265.

Aparicio, J. J., \& Huidobro, A. (1990). Las estrategias de repaso en la memoria espacial. Revista De Psicologra General y Aplicada, 43 (1), 59-69.

Bandura, A. (1984). Teorнa del aprendizaje social (2nd ed.). Madrid: Espasa-Calpe. Bicca-Marques, J. C., \& Garber, P. A. (2005). Use of social and ecological information in tamarin foraging decisions. International Journal of Primatology, 26 (6), 1321-1344. Bonnie, K. E., \& de Waal, F. B. M. (2007). Coping without rewards: socially influence foraging decisions among brown capuchin monkeys. Animal Cognition, 10, 283-292. Boyer, D., Miramontes, O., Ramos-Fernández, G., Mateos, J. L., \& Cocho, G. (2003). Modeling the searching behavior of social monkeys. Disordered Systems and Neural Networks.

Cabrera, F. (2009). Evaluando memoria de trabajo y de referencia en hámsters dorados (Mesocricetus auratus): una tarea de memoria espacial. Revista Mexicana de An6lisis de la Conducta, 35, 117-132.

De Lillo, C., Visalberghi, E., \& Aversano, M. (1997). The organization of exhaustive 
searches in a patchy space by capuchin monkeys (Cebus apella). Journal of Comparative Psychology, 111 (1), 82-90.

Fragaszy, D., Johnson Pynn, J., Hirsh, E., \& Brakke, K. (2003). Strategic navigation of two dimensional alley mazes: comparing capuchin monkeys and chimpanzees. Animal Cognition, 6, 149-160.

Fragaszy, D., Visalberghi, E., \& Fedigan, L. M. (2004). The complete capuchin: The biology of the Genus Cebus. United Kingdom: Cambridge University Press.

Heyes, C. M. (1993). Imitation, culture and cognition. Animal Behavior, 46, 999-1010. Janson, C. H. (1990). Social correlates of individual spatial choice in foraging groups of brown capuchin monkeys, Cebus apella. Animal Behaviour, 40, 910-921.

Lahitte, H. B. (1992). Modelos Estocбsticos del Aprendizaje, bases experimentales. Buenos Aires, Argentina: Ediciones Nuevo Siglo.

Sánchez Vazquez, M. J., Lahitte, H. B., \& Tujague, M. P. (2011). El análisis descriptivo como recurso necesario en ciencias sociales y humanas. Fundamentos en Humanidades, XI (II, 2010), 103-116.

Santisteban Requena, C. (2000). Modelos estocásticos de aprendizaje en ensayos de respuesta dicotómica y un número finito de estados absorbentes. Psicothema, 12 (2), 519-521.

Ludvig, N., Tang, H. M., Eichenbaum, H., \& Gohil, B. C. (2003). Spatial memory performance of freely-moving squirrel monkeys. Behavioural Brain Research, 140, 175-183.

Potì, P. (2000). Aspects of spatial cognition in capuchins (Cebus apella): frames of reference and scale of space. Animal Cognition, 3, 69-77.

Potì, P., Bartolommei, P., \& Saparoti, M. (2005). Landmark use by Cebus apella. International Journal of Primatology, 26 (4), 921-945.

Rosengart, C. R., \& Fragaszy, D. M. (2003). The role of memory in an object performance task in capuchin monkeys (Cebus apella). Journal of Primatology, 60 (1), 46-47.

Spinozzi, G., Lubrano, G., \& Truppa, V. (2002). The categorical representation of spatial relations by tufted capuchin monkeys. Folia Primatologica, 73, 297-337.

Staddon, J. E. R., \& Horner, J. M. (1989). Stochastic choice models: a comparison between Bush-Mosteller and a source -independent reward-following model. Journal of the Experimental Analysis of Behavior, 52 (1), 57-64.

Tarou, L. R., \& Maple T. L. (2000). The use of spatial memory in foraging by a group of captive golden lion tamarins (Leontopithecus rosalia). American Journal of Primatology, 51 (1), 1-20.

Tavares, M. C. H., \& Tomaz, C. (2000). Cognitive learning and declarative memory in capuchin monkeys (Cebus apella). Folia Primatologica, 71, 189-248.

Visalberghi, E., \& Addessi, E. (2001). Acceptance of novel food in capuchin monkeys: do specific social facilitation and visual stimulus enhancement play a role? Animal Behavior, 62, 567-576.

Yoerg, S. I., \& Kamil, A. C. (1982). Response strategies in the radial arm maze: running around in circles. Animal Learning \& Behavior, 10 (4), 530-534. 